\title{
Study of Propagation of Ion Acoustic Waves in Argon Plasma
}

\author{
N. S. Suryanarayana, Jagjeet Kaur, Vikas Dubey \\ Department of Physics, Govt. Vishwanath Yadav Tamaskar Post Graduate \\ Autonomous College, DURG (C.G), India \\ E-mail: nssoo2@gmail.com, jsvikasdubey@gmail.com \\ Received June 25, 2010; revised July 22, 2010; accepted August 19, 2010
}

\begin{abstract}
The properties of small amplitude acoustic waves (IAW) in unmagnetised plasma have been discussed in detail. An experimental set up to study the propagation of IAW in argon plasma has been descried. The speed of IAW under different conditions of discharge current and pressure has been measured from the time-of flight technique. From these measurements, electron temperatures have been calculated. The results have been compared with those obtained by single probe method, and were found to be in good agreement with each other so IAW speeds can be used to calculate plasma parameters.
\end{abstract}

Keywords: IAW in Argon Plasma, Study of Propagation

\section{Introduction}

Plasma can support a great variety of wave motion. Both high frequency $\left(\omega \geq \omega_{\mathrm{pe}}\right)$ and low frequency $\left(\omega \geq \omega_{\mathrm{pi}}\right)$ electromagnetic and electrostatic waves may propagate in plasma. The primary emphasis has been placed on the study of electrostatic waves because the ease with which such waves may be excited and detected and because the collision less damping of waves predicted by Landau can be conveniently studied [1-10].

Ion waves are low frequency pressure waves in plasma. If the ion plasma frequency $\left(\omega_{\mathrm{pi}}\right)$ greatly exceeds the wave frequency, both the electrons and ions oscillate almost in phase. At this stage some of the characteristics of the ion waves are similar to those of the ordinary sound waves. So they are called Ion Acoustic Waves (IAW).

The difference between ordinary Acoustic waves and Ion acoustic waves arise from the electric field induced by a slight charge separation. The electron component of the ion acoustic wave tends to propagate faster than the ion component. The electric field retards the electron motion, forcing the two species to propagate together.

Ion Acoustic waves were first predicted theoretically by Tonks \& Langmuir [11]. They were first observed, in gas discharge plasma by Rewans [12]. Landau damping in plasmas was predicted by Fried \& Gould [13] and experimentally demonstrated in a high temperature ce- sium plasma by wong et al. [14]. Ion Acoustic waves were observed in cylindrical discharge tubes by many workers [15-17] and in magnetically supported plasmas by Alesef $\&$ Neidgh [18] dispersive effects at high frequencies were studied by sessler [19] Tanaca et al. [20] and Jones et al. [21]. Ion Acoustic solutions were excited and their propagation in plasma was studied by many others [22-28]. Ion acoustic wave propagation in dusty plasmas $[29,33]$ was studied to find charge fluctuation and a new damping mechanism. The possibility of the turbulent development of an Ion Acoustic Wave that yields particle fluxes as well as energy fluxes has been used for characterization of the acoustic wave propagation in presheath region [30]. Dust acoustic solitary waves [31] and nonplaner ion acoustic waves in plasma [32] were studied by many others.

In the propagation of these ion acoustic waves (IAW), depending on the details of the velocity distribution function; energy may flow from the wave into the ions or in the opposite direction. If the phase velocity of the wave greatly exceeds the average thermal speed of the ions, the interaction is weak and energy exchange is between resonant ions and the electric field of the wave. If the phase velocity of the wave is comparable in magnitude the average ion speed, the interaction is strong and occurs by phase mixing and also by resonant exchange. The phase velocity of a small amplitude plane ion acoustic wave propagating along the field lines can be derived 
from the plasma fluid equations [34].

$$
\begin{aligned}
& \frac{\omega}{k}=C_{s}=\left[\frac{\gamma_{e} k T_{e}+\gamma_{i} k T_{i}}{m_{i}}\right]^{1 / 2} \\
& \approx\left[\frac{\gamma_{e} k\left(T_{e}+3 T_{i}\right)}{m_{i}}\right]^{1 / 2}
\end{aligned}
$$

Where $\gamma$ the ratio of specific heats, $\mathrm{k}$ is the wave number.

\subsection{Wave Excitation}

Electrostatic excitation of low frequency waves by electrodes outside the plasma is extremely inefficient because currents on the plasma periphery shield the plasma interior from the applied fields. Excitation by modulation of magnetic field is possible only if the confining field is weak and only for long wave-lenghths [35]. Various other techniques have also been reported [36,37]. In almost all experiments on externally controlled ion waves the grids are placed inside the plasma column with the plane of the grid normal to the axis of the column. This technique was introduced by Hatta et al. [38] and extensively used by wong [39] and others. The transmission of the grid is a function of the applied potential, greater negative potentials decrease the transmission. If time varying potentials are applied, it would lead to density variations. If $\omega \sim$ $\omega_{\mathrm{pi}}$, finite transit time of ions through the sheaths around the grid introduces velocity modulation [40]. Grid excitation was accomplished at frequencies $\omega<0.1 \omega_{p i}$ (7 to $130 \mathrm{KHz}$ ) for all conditions. Detection of ion acoustic waves was done either by another biased grid or with a Langmuir probe. Grid detection generally produces an improved signal-to noise ratio.

\subsection{Measuring Techniques}

Phase and amplitude measurements were made by receiving probe along the plasma column. Phase velocity has been inferred simply by measuring the time of flight of the perturbation between two fixed grids. Capacitive coupling between the grids often presents a pickup prob$l^{4} \mathrm{~m}^{41}$, especially if the exciter frequency is $>100 \mathrm{KHz}$ and the grid separation is $<1 \mathrm{~cm}$. Plasma noise is not a problem in wave experiments but to detect extremely faint signals the signal-to-noise ratio should greatly be improved.

\subsection{Wave Damping}

The most significant contribution of the work of wong et al and Alexeff et al., Y.Nakamura et al. [42] and others [43] to the experimental study of ion waves was the care- ful measurement of wave damping under conditions in which collisional damping of ion waves could be completely neglected. The collisionless damping distance was of the order of a few centimeters or much shorter than the mean free path for ion-neutral charge exchange collisions $(\lambda>1 \mathrm{~m})$.The ion drift between the grids creates a non-negligible correction to the damping rate. It is convenient to have the average damping constant. Then the characteristics of the observed ion acoustic waves appear to match closely the properties predicted for the ion acoustic wave eigenmodes. Confirmation of these results has been reported by Sato $[44]$ and others $[45,46]$.

\subsection{Effect of Collisions on Ion Acoustic Waves}

In the works of wong et al. [39] and Aleseff et al it was shown that collisions played no role in the propagation of ion acoustic waves. Collisions between ions and electrons occur frequently $\left(\tau_{e}^{-1}>3 \times 10^{6} / \mathrm{sec}\right)$ but do not affect the ion dynamics unless the electron ion energy equipartion time is comparable to $\omega^{-1}$, which does not occur unless $n>10^{13} \mathrm{~cm}^{-3} \quad \omega \sim 10^{5} / \mathrm{sec}$. Heavy particle collisions cannot be neglected. Collisions between ions do not necessarily increase the attenuation of ion waves, in fact for $T_{e} \sim T_{i}$ ion collisions decrease the damping rate because they reduce the resonant interaction [47]. Accelerated ions and the background ions would increase rather than decrease the wave damping. The effect of ion-atom collisions on ion waves was extensively studied by Anderson et al. [48]. The ion temperature could be deduced from the drop in ion acoustic wave phase velocity.

\subsection{Theoretical Considerations $[49,50]$}

A dispersion relation is obtained using fluid approximations and then a useful handy formula for the velocity of ion acoustic wave derived. Langmuir probe I-V characteristic have been used to calculate the $T_{e}$ and $n_{e}\left(n_{i}\right)$ and hence $\omega_{\mathrm{pe}}, \omega_{\mathrm{pi}}$ and $\lambda_{\mathrm{D}}$. $\omega_{\mathrm{Pe}}$ and $\omega_{\mathrm{pi}}$ are defined as Electron plasma frequency

$$
\omega_{p e}=\left[\frac{4 \pi n_{e}{ }^{2}}{m}\right]^{1 / 2}
$$

Ion plasma frequency

$$
\omega_{p i}=\left[\frac{4 \pi n_{i}^{2}}{m_{i}}\right]^{1 / 2}
$$

Since Ion Acoustic Waves propagate only below the ion plasma frequency $\omega_{\mathrm{pi}}\left(\omega<<\omega_{\mathrm{pi}}\right)$ both electrons and ions move together. If $T_{e}>T_{i}$, the ion wave is not strongly damped. Integrating equation of motion for 
electrons, we get the Boltzmann relation.

$$
n_{e}=n_{0} \exp \left(e \phi / k T_{e}\right) \cong n_{0} \exp \left(1+e \phi / k T_{e}\right)
$$

Ion acoustic perturbation follow

$$
\frac{\partial^{2} n_{i}}{\partial t^{2}}-C_{s}^{2} \frac{\partial^{2} n_{i}}{\partial x^{2}}-\lambda_{D}^{2} \frac{\partial^{4} n_{i}}{\partial x^{2} \partial t^{2}}=0
$$

Where $\mathrm{C}_{\mathrm{s}}=(\mathrm{Te} / \mathrm{M})^{1 / 2}$ and $\lambda_{\mathrm{D}}=\left(T_{e} / 4 \pi n_{e}\right)^{1 / 2}$ is the ion acoustic speed and Debye length. By solving the above equation the dispersion relation is obtained

$$
\omega^{2}=\frac{C_{s}^{2} k^{2}}{1+k^{2} \lambda_{D}{ }^{2}}
$$

If $\mathrm{k}^{2} \lambda_{\mathrm{D}}^{2}<<1$; phase velocity of IAW equals the $\mathrm{c}_{\mathrm{s}}$ and is constant and if $\mathrm{k}^{2} \lambda_{\mathrm{D}}{ }^{2} \sim 1$ the wave becomes dispersive. The handy formula for $\mathrm{C}_{\mathrm{s}}$ is

$$
\frac{\omega}{k}=C_{s}=\frac{\left(k T_{e}+\gamma_{i} k T\right)_{i}}{m} \cong\left[\frac{\gamma k\left(T_{e} 3 T_{i}\right)}{m}\right]^{1 / 2}
$$

\subsection{Single Langmuir Probe}

A small electrode is inserted in to the plasma and the current is measured as a function of the applied voltage. The voltage is measured with respect to some convenient reference point, which is often the cathode of the discharge. The log I - V characteristic is shown in Figure 2. The plasma potential $\left(\mathrm{V}_{\mathrm{p}}\right)$ is obtained. Another potential, which is shown as $V_{f}$, is the floating potential is also obtained. Let us consider a perfectly reflecting probe. If the electrons have a Maxwellian distribution the Boltzmann's relation can be used

$$
n_{e}=n_{0} \exp \left(e V / k T_{e}\right)
$$

Thus as we approach the probe, electron density decreases. Let us consider now an absorbing probe. When the mean free path is large compared with the size of the probe, the number hitting the absorbing probe is essentially the same. Thus the electrons will move to the probe from regions much further away than the plasma boundary without making a collision. But as the probe absorbs the electrons the local electron density is depleted with an absorbing probe the current density is given by

$$
I=I_{0} \exp \left(\frac{e V}{k T_{e}}\right)
$$

Taking logarithm on both sides, the equation is

$$
\log I=\log I_{0}+\left(e V / k T_{e}\right)
$$

Thus the slope of the plot of log I (Vs) V shown in Figure 2 gives the electron temperature. Location of the knee of the curve gives the plasma potential. The other handy formulas used to calculate the plasma frequency $\omega_{\text {pe }}$ and Debye length $\lambda_{\text {De }}$ are

$$
\begin{gathered}
\omega_{p e}=\left[\frac{4 \pi n_{e}^{2}}{m}\right]^{1 / 2} \\
f_{p}=9000(n)^{1 / 2} / \mathrm{sec} \text {. or }
\end{gathered}
$$

Where $\mathrm{n}=$ number of electrons per $\mathrm{cm}^{3}$.

$$
\begin{aligned}
& \lambda_{D e}=\left[\frac{k T_{e}}{4 \pi n_{e}{ }^{2}}\right]^{1 / 2} \\
& \lambda_{D e}=6.9(T / n)^{1 / 2}
\end{aligned}
$$

\section{Experimental Setup}

The experimental set up has been shown in Figure 1. The dimensions of the vacuum chamber are $30 \mathrm{~cm}$ diameter; $50 \mathrm{~cm}$ length of stainless steel. First it was evacuated to a pressure of $2 \times 10^{-5}$ torr. The experiment was performed in the pressure range of $2 \times 10^{-4}$ to $3 \times$ $10^{-3}$ torr. of Argon. Argon was flown continuously for half an hour before the production of plasma. 10 filaments of Tungsten ( $4 \mathrm{~cm}$ length and $0.05 \mathrm{~cm}$ diameter) were symmetrically distributed and fixed to the two Aluminium supporting rings. First they were heated through supplying heater current $(\approx 1.5$ Amp. max. per filament). The discharge was created by applying the voltage between the heated filaments and wall of the chamber. The discharge current was varied between 0.75 Amps. to 0.075 Amps. So the plasma was quiescent. Plasma was spread into the chamber symmetrically. The potential across the filament was $4 \mathrm{~V}$. The discharge voltage was around 60 volts.

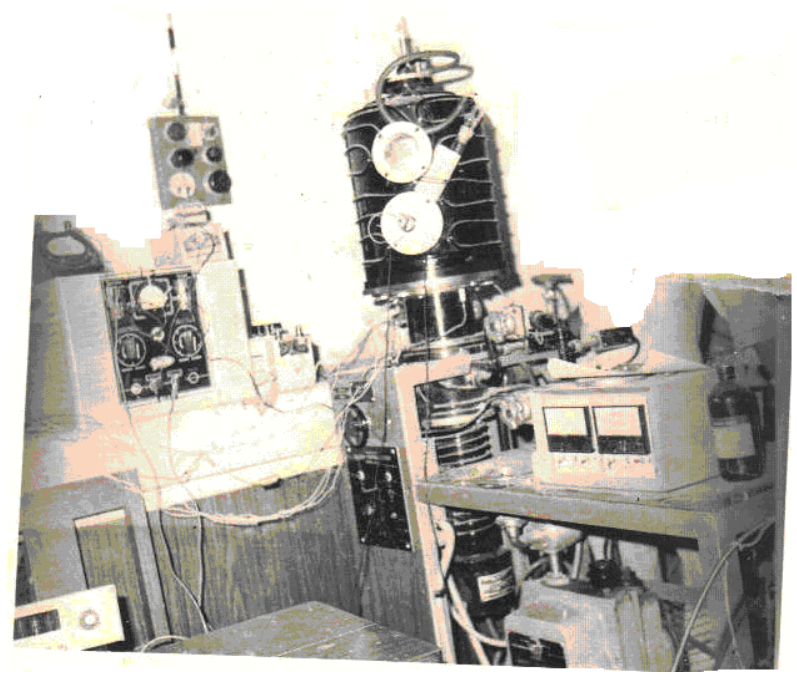

Figure 1. Photograph of experimental setup. 


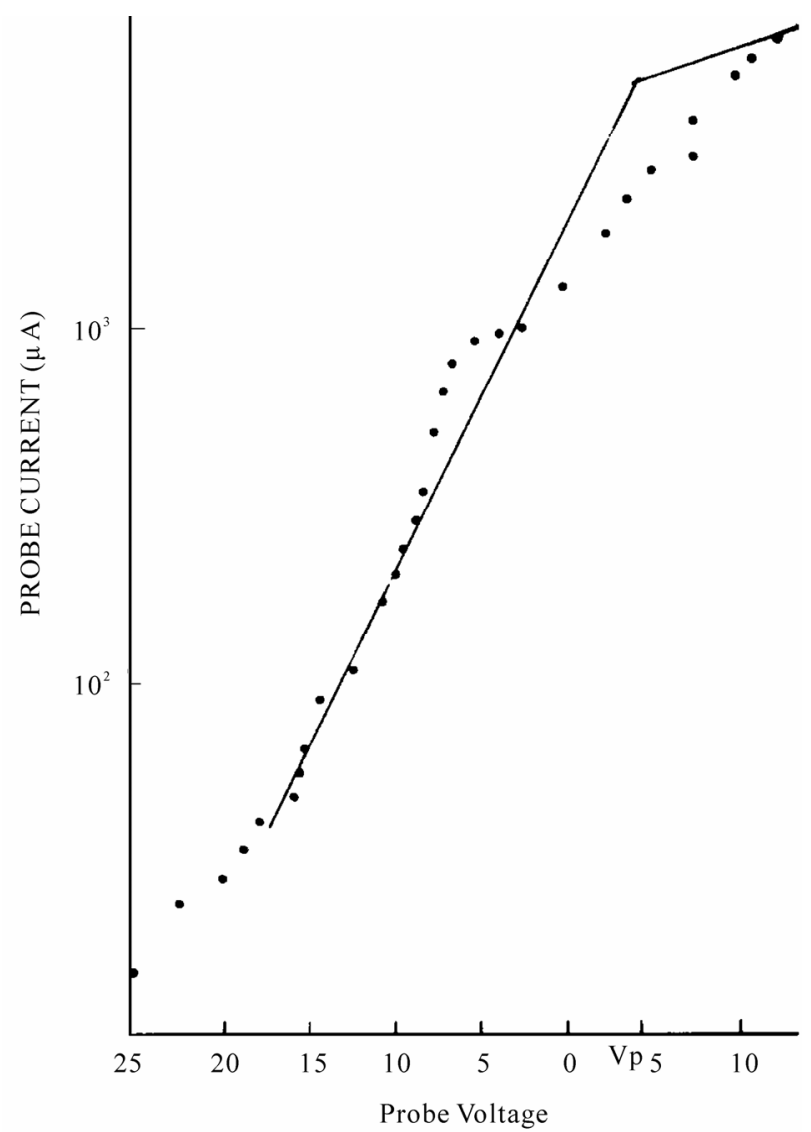

Figure 2. Log I-V Curve of langmuir probe.

A $1 \mathrm{~cm}$ diameter thin aluminium disk was used as the Langmuir probe. The probe voltage was varied between $-70 \mathrm{~V}$ to $+25 \mathrm{~V}$. The probe current has been measured by a Keithly multimeter.

For the launching of Ion Acoustic waves a $30 \mathrm{KHz}$ pulse from the pulse generator was applied to the negatively biased transmitting probe. The biasing can also be floating. This probe was situated at the centre of the plasma and normal to the axis of the chamber and it was fixed in the chamber through a feed through.

For collecting the ion acoustic waves, the Langmuir probe was used. It was biased positive to collect the electrons (electron current). The voltage developed at the ends of $1 \mathrm{k} \Omega$ resistance was given to the CRO $\mathrm{Y}_{1}$ terminal, through a $100 \mathrm{PF}$ capacitor. The pulse, directly from pulse generator was fed to the $\mathrm{Y}_{2}$ of CRO. Both the traces were locked through the adjustment of H. F. sync. and level controls of CRO. The received voltage was in the $20 \mathrm{mV}$ to $50 \mathrm{mV}$ range. While the transmitted voltage was $<1 \mathrm{~V}$.

\section{Observations \& Results}

Table 1 presents a comprehensive list of $T_{e}$ (electron temperature) calculated through (1) Ion Acoustic wave and (2) By single Langmuir probe for various discharge current at various operating pressures.

1) a) Probe current was obtained at different Langmuir probe voltages and discharge currents at three different pressures.

b) A sample log $\mathrm{I}-\mathrm{V}$ characteristic of Langmuir probe has been shown in Figure 2.

c) The electron density/ion density, electron temperatures, plasma frequency, plasma ion frequency, Debye length were calculated from log $\mathrm{I}-\mathrm{V}$ characteristics.

2) The traces of the transmitted wave and received ion acoustic wave, appearing on the CRO screen, were carefully drawn by putting a transparent paper on the CRO screen, at different probe separations, for varying discharge currents and pressures. Time taken by ion acoustic wave to travel from transmitting grid to receiving grid was obtained by the time of the flight techniques. Velocity of the IAW was calculated and tabulated.

3) Electron temperature was calculated for different sets of readings.

4) The electron temperature obtained by Langmuir probe method was compared with that obtained from the time of - flight techniques.

\section{Discussion and Conclusions}

1) The calculation of electron temperature by IAW propagation studies Figure 3 to Figure 8 and by Langmuir probe techniques Figure 1 indicate that the two values are in fair agreement. However $\mathrm{T}_{\mathrm{e}}$ calculated from Langmuir probe techniques is slightly more ( 10 to $15 \%)$ than that calculated by IAW propagation speeds. Langmuir probe suffers from a number of defects such as-

a) Laboratory plasma deviates from some of the assumptions made, while developing theory, such as Maxwellian distribution of energy and velocity, isotropy of plasma, homogeneity and quasinutrality of plasma.

b) Probe Size: It is assumed that the presence of probe does not change the distribution and that the size of the probe is negligible in comparison with reference electrode. But finite probe size always as important role and disturbs the distribution.

c) Sheath: In general a transition region called sheath appearing around the probe, is assumed to be independent of probe potential. But at strong negative probe potentials the central part of the sheath has increased electron density due to the presence of repelled electrons from the probe and incoming electrons to the probe i.e. the energy distribution near the probe may not be equal to that existing in undisturbed plasma. Further, probe current is also affected by secondary emission from probe by the action of photons, ions and metastable atoms. 
Table 1. A comprehensive list of $T_{e}$ (electron temperature) calculated by (1) Ion Acoustic wave and (2) By single Langmuir probe for various discharge current at various operating pressures.

\begin{tabular}{|c|c|c|c|c|c|c|}
\hline \multicolumn{3}{|c|}{ Gas used = Argon } & \multicolumn{4}{|c|}{ Temperature $=306 \mathrm{~K}$} \\
\hline S.No. & Pressure in torr & $\begin{array}{l}\text { Discharge } \\
\text { Current Amp }\end{array}$ & $\begin{array}{l}\text { Velocity of IAW by } \\
\text { time-of-flight measurement } \\
\times 10^{5} \mathrm{~cm} / \mathrm{sec} \text {. }\end{array}$ & $\begin{array}{l}\text { Te obtained by } \\
\text { velocity of IAW } \\
(\mathrm{eV})\end{array}$ & $\begin{array}{l}\text { Te obtained by } \\
\text { Langmuir probe } \\
(\mathrm{eV})\end{array}$ & Ion temperature \\
\hline 1 & $2.5 \times 10^{-4}$ & 0.5 & 2.96 & 3.65 & 4.3 & \multirow{16}{*}{$\sim 800 \mathrm{~K}$} \\
\hline 2 & $6.5 \times 10^{-5}$ & 0.5 & 2.34 & 2.27 & - & \\
\hline 3 & $1 \times 10^{-3}$ & 0.5 & 2.14 & 1.90 & 2.1 & \\
\hline 4 & $6 \times 10^{-4}$ & 0.35 & 2.6 & 2.84 & 3.5 & \\
\hline 5 & $2.5 \times 10^{-3}$ & 0.35 & 1.95 & 1.59 & - & \\
\hline 6 & $1 \times 10^{-3}$ & 0.35 & 2.03 & 1.72 & - & \\
\hline 7 & $1.5 \times 10^{-4}$ & 0.35 & 2.78 & 3.23 & \multirow{3}{*}{1.5} & \\
\hline 8 & $3 \times 10^{-4}$ & 0.11 & 1.82 & 1.38 & & \\
\hline 9 & $7 \times 10^{-4}$ & 0.11 & 1.67 & 1.16 & & \\
\hline 10 & $2.5 \times 10^{-3}$ & 0.11 & 1.54 & 0.99 & - & \\
\hline 11 & $3 \times 10^{-3}$ & 0.075 & 1.43 & 0.85 & 0.8 & \\
\hline 12 & $3 \times 10^{-3}$ & 0.2 & 1.46 & 0.89 & - & \\
\hline 13 & $3 \times 10^{-4}$ & 0.2 & 2.27 & 2.15 & - & \\
\hline 14 & $2.5 \times 10^{-3}$ & 0.7 & 2.83 & 3.34 & - & \\
\hline 15 & $6 \times 10^{-4}$ & 0.75 & 2.84 & 3.37 & - & \\
\hline 16 & $2.5 \times 10^{-4}$ & 0.75 & 2.84 & 3.37 & 4.0 & \\
\hline
\end{tabular}

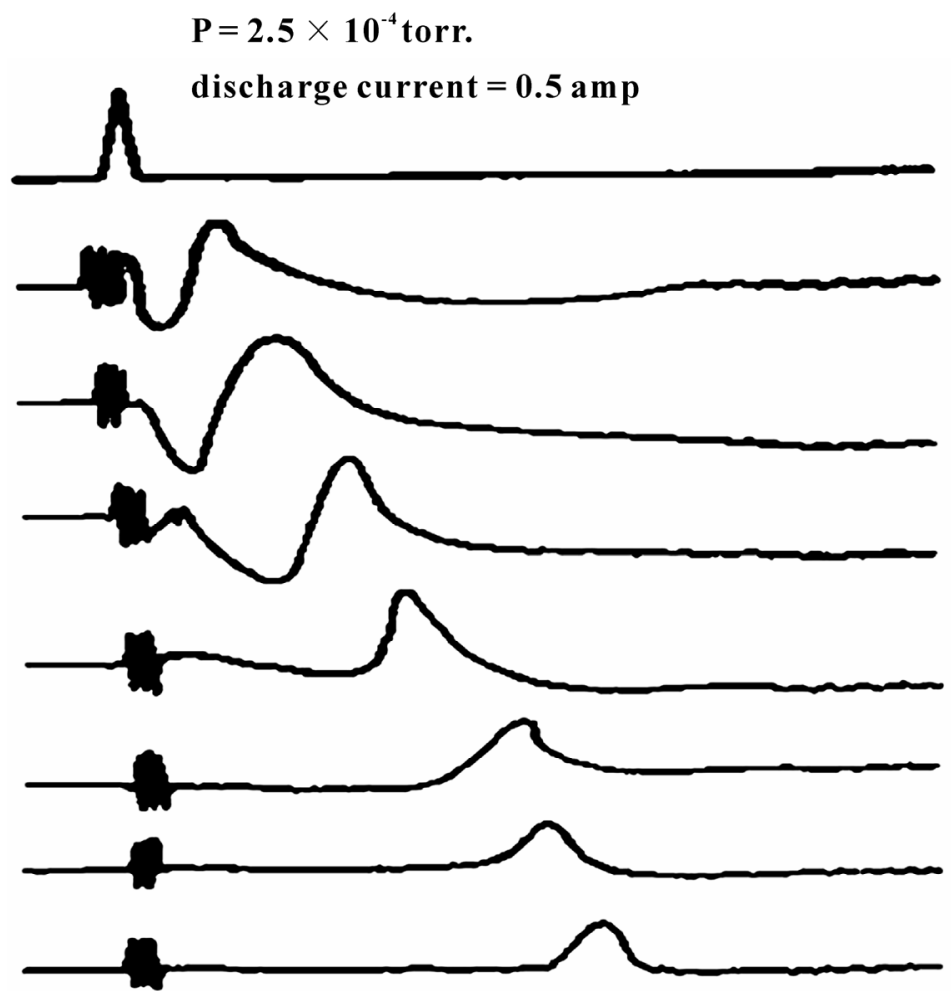

\section{Probe Seperation}

\section{Launching Pulse}

0.75 c.m.

1.1 c.m. Detected pulse at probe seperation

1.8 c.m. Detected pulse at probe seperation

2.5 c.m. Detected pulse at probe seperation

3.4 c.m. Detected pulse at probe seperation

3.9 c.m.

Detected pulse at probe seperation

4.3 c.m. Detected pulse at probe seperation

Figure 3. Trace for launching pulse and detected pulse by moving probe in various distance (discharge current $=0.5$ amp). 


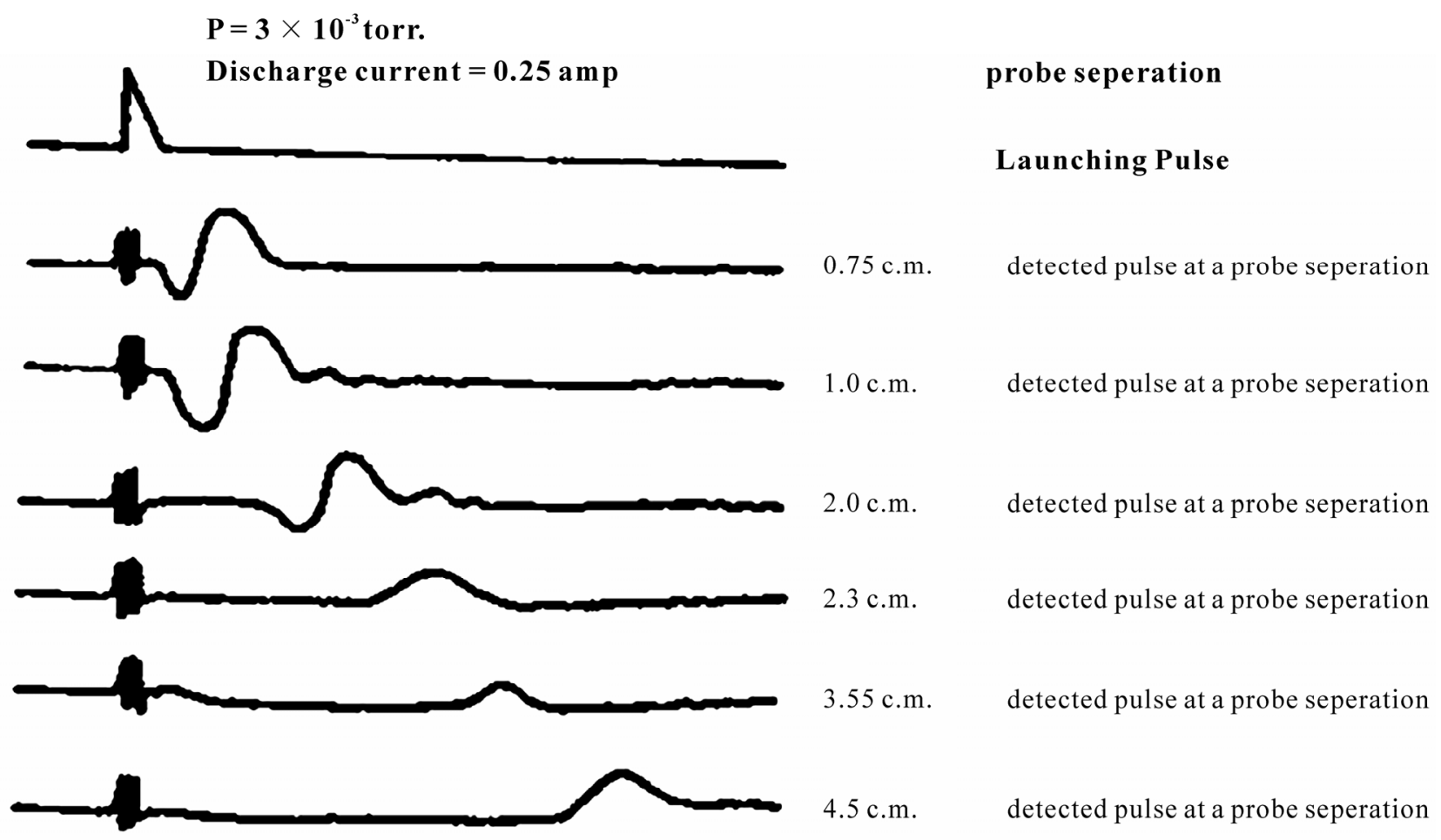

Figure 4. Trace for launching pulse and detected pulse by moving probe in various distance (discharge current $=0.25$ amp).

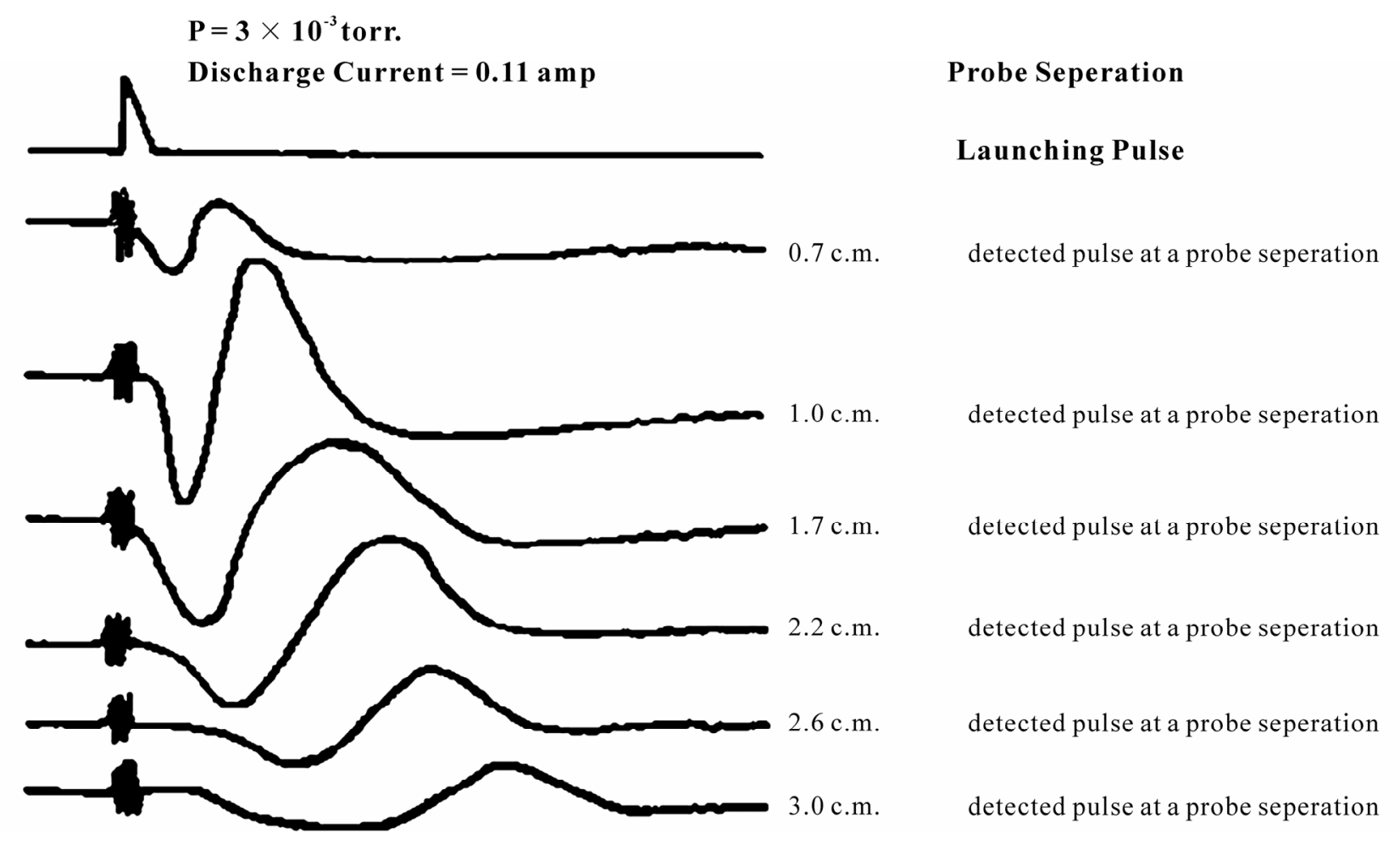

Figure 5. Trace for launching pulse and detected pulse by moving probe in various distance (discharge current $=0.11$ amp). 


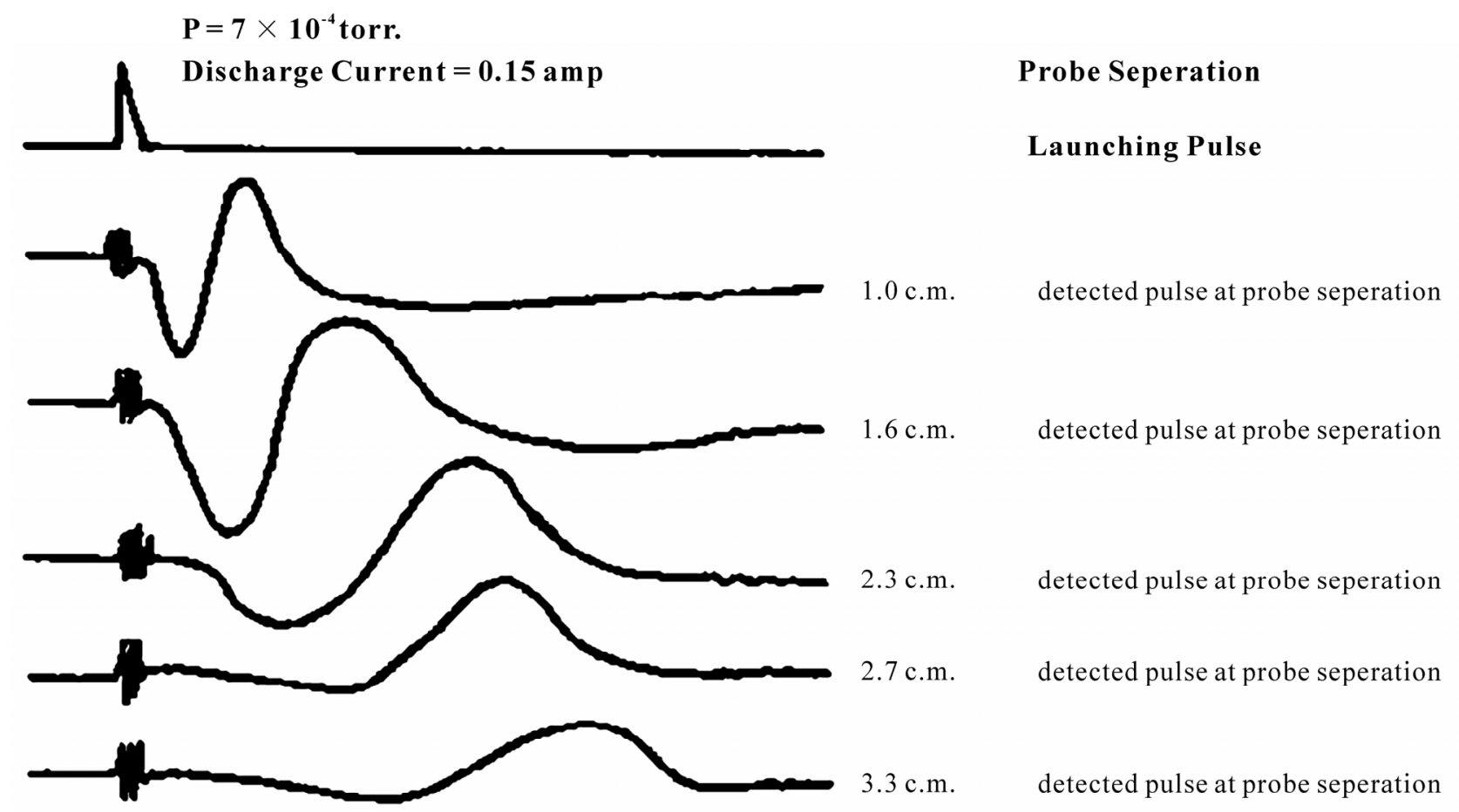

Figure 6. Trace for launching pulse and detected pulse by moving probe in various distance (discharge current $=0.15$ amp).

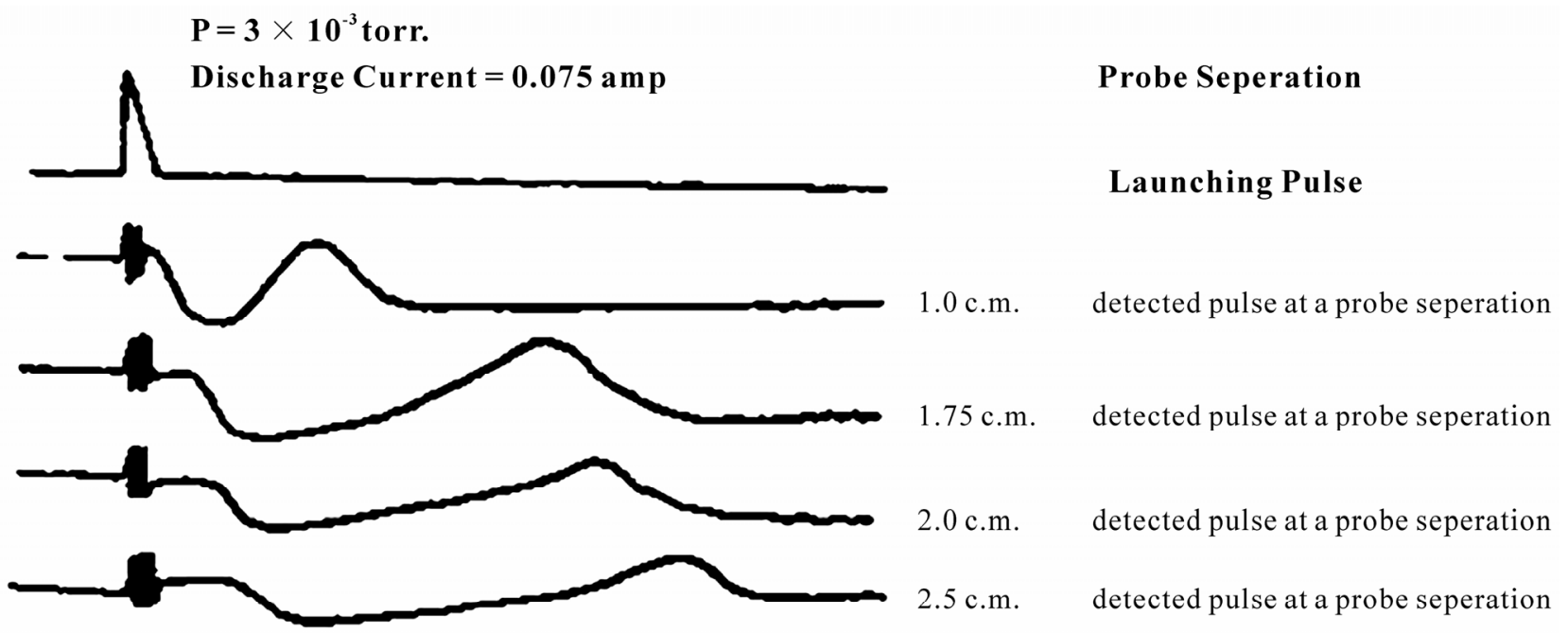

Figure 7. Trace for launching pulse and detected pulse by moving probe in various distance (discharge current $=0.075$ amp).

d) In the measurements: The probe potential as measured by an instrument may not represent the true potential difference between the electrodes and probe. There may be contract potentials. A part of the voltage applied to the probe is dropped across the sheath and it is an unknown quantity to the experiments. The space potential Vs may vary due to the presence of fluctuations and drift with in the bulk plasma and more intrinsically by the driving probe current through the plasma without infinite conductivity.

e) The equivalent plasma resistance, $R$, between probe and reference electrode plays a significant role on the determination of Te. $\mathrm{R}$ in the measuring circuit of the probe depends on both the plasma conductivity and configuration of the probe.

f) Probe surface contamination also plays an important 


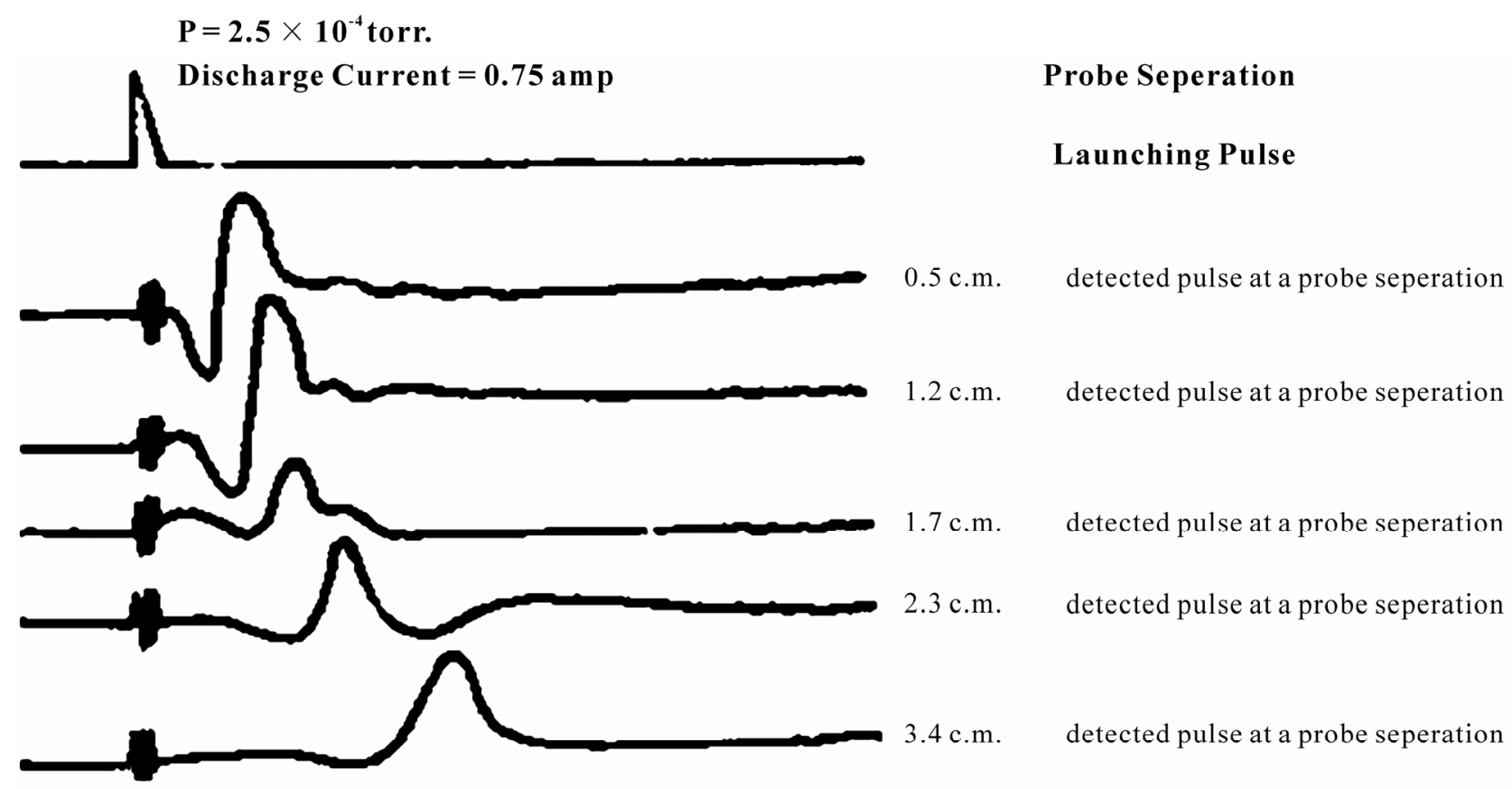

Figure 8. Trace for launching pulse and detected pulse by moving probe in various distance (discharge current $=0.75$ amp).

role, which can not be avoided.

IAW speed determination involves Time-of-Flight measurements. Each small division on the CRO screen represents $1 \mu$ s (in present case). Measurement of time can be in an error of $\pm 0.5 \mu$ s i.e. the determination of speed may be in error by about 5 to $10 \%$. Moreover, we have neglected ion temperature in comparison with electron temperature, taking an electron temperature of the order of $30,000 \mathrm{~K}$ and $3 \mathrm{~T}_{1}$ of the order of $1500 \mathrm{~K}$. so it also involves an error of $5 \%$ we can therefore argue that the determination by the two different techniques are in good agreement.

2) When an IAW is excited by applying a signal, either a voltage pulse, or sinusoidal wave is applied to the grid, a receiver detects not only a signal of the wave but also a direct coupled signal. This is evident from the various oscillogram presented. The coupling was thought to be [54] a capacitive coupling between the transmitter and the detector. In fact Nakamura et al. [42] has shown that it is associated with the change of plasma potential, caused by drawing of electrons by grids and probes.

3) The oscillogram presented in this case shows that the amplitude of IAW goes down or diminishes as it travels longer distances. This is due to damping (collisional, non-collisional).

4) Our observations show that when the distance between the transmitting grid and the receiving probe is small, Figure 3 to Figure 8 the speed of IAW is large, which we have neglected in taking the average. It is quite reasonable that when the grid and the receiving probe are close by, the electric field is strong, thereby enhancing the velocity of electrons reaching the probe. This enhances the electron temperature in the neighborhood of the transmitting grid, which in turn increases the speed of IAW.

\section{References}

[1] T. H. Stix, “The Theory of Plasma Waves,” McGarw Hill, New York, 1962.

[2] V. L. Ginsberg, "The Properties of Electromagnetic Waves in Plasmas,” 2nd Edition, Pergamon, Oxford, 1970.

[3] T. J. M. Boyd and J. J. Sanderson, "Plasma Dynamics,” Berns \& Nobel, New York, 1969.

[4] J. L. Shoket, "The Plasma State,” Academic Press, New York, 1971.

[5] N. A. Krall and A. W. Trivelpiece, "Principle of Plasma Physics,” McGraw Hill, New York, 1973.

[6] G. M. Sessler, "Physical Acoustics, Vol. IVB,” Vol. 99, 1968.

[7] I. Alexeff, W. D. Jones and D. Montgomery, Proceedings of Conference on Physics of Quiscent Plasmas, Tresti Italy (Associazione Eurat on - CNEN, Rome), Vol. 2, 1967, p. 371.

[8] I. Alexeff, W. D. Jones and M. G. Payne, Bulletin of the American Physical Society, Vol. 11, 1966, p. 843.

[9] I. Alexeff and W. D. Jones, Applied Physical Letters, Vol. 99, 1966, p. 77.

[10] I. Alexeff, W. D. Jones and D. Montgomery, Bulletin of the American Physical Society, Vol. 12, 1967, p. 711. 
[11] L. Tonks and I. Langmuir, Physical Review, Vol. 33, 1929, p. 195.

[12] R. W. Revans, Physical Review, Vol. 44, 1933, p. 798.

[13] B. D. Fried and R. W. Gould, Physics of Fluids, Vol. 4, 1961, p. 139.

[14] A. Y. Wong, R. W. Motley and N. D’Angelo, Physical Review, Vol. 133, 1964, p. A436.

[15] Y. Hatta and N. Sato, Proceedings of 5th International Conference on Ionization Phenomenon in Gases, $\mathrm{H}$. Maecker, Ed., North Holland Publishing Co., Amsterdam, Vol. 1, 1962, p. 478.

[16] F. W. Crawford and S. A. Self, Proceedings of 6th International Conference on Ionization Phenomenon in Gases, P. Hubert and Cremeien Bureau des., Ed., Paris, Vol. 3, 1964, p. 129.

[17] P. J. Barrett and P. F. Little, Physical Review Letter, Vol. 14, 1965, p. 365.

[18] I. Alexeff and R. V. Neidigh, Physical Review, Vol. 129, 1963, p. 516.

[19] G. M. Sessler, Physical Review Letters, Vol. 17, 1966, p. 243.

[20] H. Tanaca, M. Koganei and A. Hirose, Physical Review Letters, Vol. 16, 1966, p. 1079.

[21] W. D. Jones and I. Alexeff, Proceedings of 7th International Conference on Ionization Phenomenon in Gases, Gradevinskknija, Yugoslavia, Vol. 2, 1966, p. 330.

[22] H. Ikezi, R. J. Taylor and D. R. Baker, Physical Review Letters, Vol. 25, 1970, p. 11.

[23] R. J. Taylor, K. R. Mackenzie and H. Ikezi, Review of Scientific Instruments, Vol. 43, 1972, p. 1675.

[24] H. Ikezi, Physics of Fluids, Vol. 10, 1973, p. 1668.

[25] M. Q. Tran, Physica Scripta, Vol. 20, 1979, p. 317.

[26] Y. Nakamura and T. Ogino, Plasma Physics, Vol. 24, 1982, p. 1295.

[27] Y. Nakamura, IEEE Transactions on plasma science PSVol. 10, 1982, p. 180.

[28] S. Watanabe, Journal of Plasma Physics, Vol. 14, 1975, p. 353.

[29] J.-J. Li, D.-L. Xiao, Y.-F. Li and J.-X. Ma, Chinese Physical Letters, Vol. 24, 2007, p. 151.

[30] U. Deka, C. B. Dwivedi and H. ramchandran, Physica Scripta, Vol. 73, 2006, p. 87.

[31] A. P. Mishra and A. R. Chowdhury, Physical Plasmas, Vol. 13, 2006, pp. 62307-62314.

[32] A. P. Mishra and C. Bhowmik, Physical Letters A, Vol. 369, 2007, pp. 90-98.

[33] A. P. Mishra, H. Bailung and J. Chutia, Physical Plasmas,
Vol. 17, 2010, pp. 44502-44505.

[34] L. Landau, Journal of Physics, Vol. 10, 1946, p. 25.

[35] L. Spitzer, “The Physics of Fully Ionized Gases,” Wiley Inter science, New York, 1962.

[36] P. F. Little, Proceedings of 5th International Conference Ionization Phenomenon in Gases, North Holland Publishing Co., Amsterdam, 1962

[37] J. M. Buzzi, H. J. Doucet and W. D. Jones, Proceedings of 3rd International Conference on Quiscent Plasmas Elsinore, Gjellerup, Copenhagen, 1971.

[38] R. N. Franklin, G. J. Smith, S. M. Hamberger and G. Lampis, Physical Letters A, Vol. 36, 1971, p. 473.

[39] H. Ikezi, N. takahashi and K. Nishikawa, Physics of Fluids, Vol. 12, 1969, p. 853.

[40] A. Y. Wong and D. R. Baker, Physical Review, Vol. 188, 1969, p. 326.

[41] I. B. Bernstein, J. L. Hirschfield and J. H. Jacob, Physics of Fluids, Vol. 14, 1971, p. 628

[42] Y. Nakamura and Y. Nomura, Physical Letters A, Vol. 65, 1978, p. 415.

[43] Y. Nakamura, Y. Nomura and K. E. Lonngren, Research Note, ISAS, RNO of Tokyo.

[44] L. Scott, Review of Scientific Instruments, Vol. 51, 1980, p. 383.

[45] N. Sato, H. Ikezi, Y. Takanashi and Yamashita, Physical Review, Vol. 183, 1969, p. 278.

[46] J. F. Wendt and A. B. Cambel, Proceedings of International Conference on Physics of Quiescents, Plasmas Frascati, Lab Gas Ionization Rome, 1967.

[47] A. Y. Wong, Applied Physical Letters, Vol. 6, 1965, p. 147.

[48] R. W. Motley and A. Y. Wong, Proceedings of 6th International Conference on Ionization Phenomena in Gasesbureau des. Editions, France, 1963, Vol. 3, p. 133.

[49] H. K. Andersen, N. D. Angelo, V. O. Jensen, P. Michelsen and P. Nielsen, Physics of Fluids, Vol. 11, 1968, p. 1177.

[50] H. Ikezi, "Solitons in Action,” K. E. Lonngren and A. Scott, Ed., Academic Press, New York, 1978, p. 153.

[51] F. F. Chen, "Introduction to Plasma Physics," Plemun Press, New York, 1974.

[52] J. E. Allan, Plasma Physics, B. E. Keen, Ed., Conference Series, Institute of Physics, London, 1974, p. 131.

[53] J. D. Swift and M. J. R. Schwar, "Electrical Probs for Plasma Diagnostics,” Iliffe, London, 1969.

[54] I. Alexeff, W. D. Jones, K. Lonngren and D. Montgomery, Physics of Fluids, Vol. 12, 1969, p. 345. 